\title{
Herschel Planetary Nebula Survey (HerPLaNS): Construction of a Detailed Dusty Photoionization Model of NGC6781
}

\author{
Masaaki Otsuka ${ }^{1}$, Toshiya Ueta ${ }^{2}$, You-Hua Chu ${ }^{1,3}$, Kentaro Asano ${ }^{2}$ \\ and HerPLaNS consortium \\ ${ }^{1}$ ASIAA, 11F of Astronomy-Mathematics Building, AS/NTU. No.1, Sec. 4, Roosevelt Rd, \\ Taipei 10617, Taiwan, R.O.C. email: otsuka@asiaa.sinica.edu.tw (MO) \\ ${ }^{2}$ Department of Physics and Astronomy, University of Denver, 2112 E. Wesley Ave., Denver, \\ CO 80210, USA \\ ${ }^{3}$ Department of Astronomy, University of Illinois, 1002 West Green Street, Urbana, Illinois, \\ USA
}

\begin{abstract}
As one of the follow-up studies of Herschel Planetary Nebula Survey (HerPlaNS; Ueta et al. 2014), we focus on a bipolar planetary nebula (PN) NGC6781 to characterize the dusty nebula and the central star based on our own Herschel data and the rich archival spectroscopic/photometric image data in the wavelengths from UV to far-IR. With CLOUDY, we constructed a comprehensive photoionization model of NGC6781 ever made including data from UV to radio. We succeeded to reproduce the observed spectral energy distribution (SED) and the atomic gas, $\mathrm{H}_{2}, \mathrm{CO}$, and $\mathrm{OH}$ molecular line fluxes. We found that about $40 \%$ of the total dust mass would be from warm-cold dust components.
\end{abstract}

Keywords. infrared: ISM, ISM: abundances, dust, molecules, planetary nebulae: individual (NGC6781)

\section{Results}

We performed a comprehensive analysis of the PN NGC6781 in order to investigate the physical conditions of each of the ionized, atomic, and molecular gas components as well as the dust component in the nebula and the evolution of the central star, based on our own far-IR Herschel data (Ueta et al. 2014) and the rich archival spectroscopic/photometric data in the UV to far-R wavelength ranges.

Spitzer/IRS mid-IR spectrum shows the polycyclic aromatic hydrocarbon (PAH) 6$9 \mu \mathrm{m}$ and $11.3 \mu \mathrm{m}$ bands and pure rotational hydrogen molecule $\left(\mathrm{H}_{2}\right)$ lines. The excitation curve plots for the $\mathrm{H}_{2}$ lines can be fitted by a single temperature of $1050 \mathrm{~K}$. We derived the column density $N\left(\mathrm{H}_{2}\right)=9.12 \times 10^{18} \mathrm{~cm}^{-2}$. Comparison of the observed $\mathrm{H}_{2}$ line intensities relative to the $\mathrm{H}_{2} v=0-0 \mathrm{~S}(2)$ at $9.67 \mu \mathrm{m}$ line with the theoretical shock models by Flower \& Pineau Des Forêts (2010) suggests that the $\mathrm{H}_{2}$ lines are excited by shock interaction with the remnant asymptotic giant branch (AGB) circumstellar envelope.

We performed detailed plasma diagnostics and derived nebular abundances of the nine elements. By comparing with the AGB nucleosynthesis model of Karakas (2010), we found that the progenitor would be a 2.25-3.0 $M_{\odot}$ star. The current evolutional status and nebular elemental abundances of the $\mathrm{H}_{2}$-rich bipolar PN NGC6720 (e.g., van Hoof et al. 2010) are in excellent agreement with NGC6781, suggesting that both PNe have evolved from a very similar mass star and share with their evolution.

We constructed the photoionization model with CLOUDY (Ferland et al. 2013) to be self-consistent with all the observations (Fig. 1). About $40 \%$ of the total dust mass 


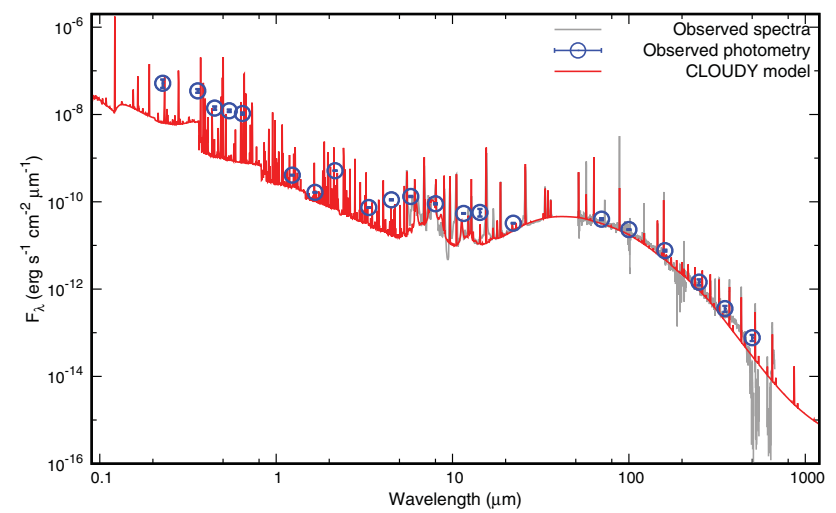

Figure 1. The full SED of the best-fit CLOUDY model of NGC6781 (red line; spectral resolution $(R)=300$ ), compared with the observational constrains: photometry data (blue circles) and spectroscopy data (grey line).
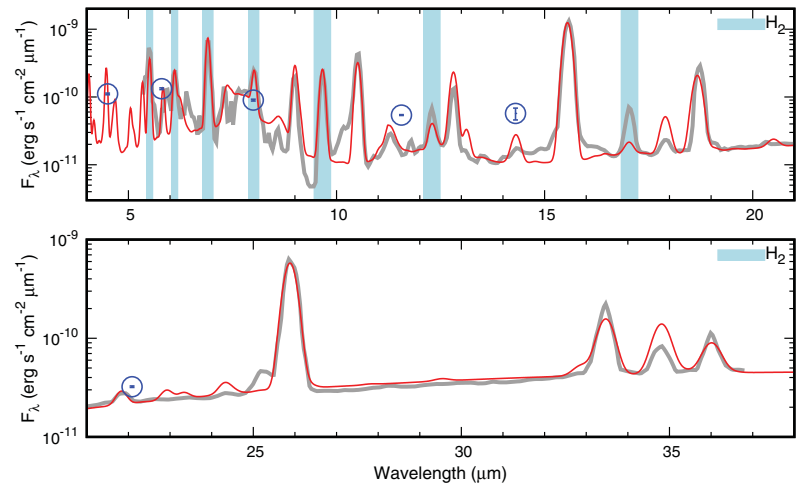

Figure 2. Comparison between the observed data (grey line and blue circles) and the best-fit CLOUDY model (red line; $R=100$ ). The positions of the $\mathrm{H}_{2}$ lines are highlighted.

$2.13 \times 10^{-3} M_{\odot}$ would be from warm-cold dust components. The derived gas mass of $0.58 M_{\odot}$ corresponds to $\sim 70 \%$ of the total mass loss in initially $3.0 M_{\odot}$ stars predicted by Karakas (2010). We found that additional heating sources are necessary to explain the observed $\mathrm{H}_{2}$, $\mathrm{CO}$, and $\mathrm{OH}$ line fluxes. By introducing warm regions with a constant kinematic gas temperature of $\sim 1000 \mathrm{~K}$ in the photodissociation region (PDRs), we succeeded to reproduce these molecule line fluxes (Fig. 2).

To verify physical conditions of the warm regions in the PDRs introduced in our model, atomic gas and molecule emission line maps are necessary. A wide variety of molecular gas abundances are essential to further characterize the PDR conditions in NGC6781 and obtain more exact elemental abundances. A more detailed discussion will be found in Otsuka et al. (in prep).

\section{References}

Ferland, G. J., Porter, R. L., van Hoof, P. A. M., et al. 2013, RMxAA, 49, 137

Flower, D. R. \& Pineau Des Forêts, G. 2010, MNRAS, 406, 1745

Karakas, A. I. 2010, MNRAS, 403, 1413

Ueta, T., Ladjal, D., Exter, K. M., et al. 2014, A\&A, 565, A36

van Hoof, P. A. M., van de Steene, G. C., Barlow, M. J., et al. 2010, A\& A, 518, L137 